\title{
Microstructure of the Pulsed Laser Deposited LaSrCuO Films
}

\author{
M.Z. Cieplak ${ }^{a}$, A. Abal'oshev ${ }^{a}$, I. Zaytseva ${ }^{a}$, \\ M. Berkowski ${ }^{a}$, S. GUhA ${ }^{b}$ AND Q. WU ${ }^{b}$ \\ ${ }^{a}$ Instytut Fizyki Polskiej Akademii Nauk \\ al. Lotników 32/46, 02-668 Warszawa, Poland \\ ${ }^{b}$ Department of Physics and Astronomy, Rutgers University \\ Piscataway, NJ 08851, USA
}

The X-ray diffraction and atomic force microscopy are used to examine the microstructure of $\mathrm{La}_{1.85} \mathrm{Sr}_{0.15} \mathrm{CuO}_{4}$ films grown by pulsed laser deposition on $\mathrm{LaSrAlO}_{4}$ substrates. The films grow with different degrees of built-in strain, ranging from a large compressive to a large tensile in-plain strain. The tensile strain cannot be attributed to a substrate-related strain. The possible origins of the tensile strain are discussed.

PACS numbers: 68.55.-a, 68.55.Jk, 68.37.Ps

\section{Introduction}

The properties of epitaxial films depend on the strain induced by the lattice parameters mismatch between the substrate and the film. The example is the substrate-induced compressive in-plane strain in $\mathrm{La}_{1.85} \mathrm{Sr}_{0.15} \mathrm{CuO}_{4}$ (LSCO) films which enhances superconducting transition temperature, $T_{\mathrm{c}}$ [1]. The thick LSCO films grown by various methods under similar conditions show film-to-film variation of many properties. It has been suggested that the substrate-related strain is responsible for this effect $[2,3]$. In this study we examine the microstructure of LSCO films (of a thickness of about $5000 \AA$ ) grown by pulsed laser deposition (PLD) on single-crystalline $\mathrm{LaSrAlO}_{4}$ (LSAO) substrates. We use X-ray diffraction and atomic force microscopy (AFM) to determine the structure and morphology of the films. The substrate in-plane lattice parameter, equal to $3.756 \AA$, is about $0.5 \%$ smaller than the in-place lattice parameter of LSCO which is equal to $3.777 \AA$. As a result, a compressive in-plane strain may be expected. Surprisingly, our study indicates that some films grow with the tensile strain, which cannot be attributed directly to the influence of the substrate. 


\section{Experimental details, results, and discussion}

The parameters of the deposition process were optimized prior to this study [4]. The $c$-axis lattice parameters were determined from eight high-angle $(00 l)$ diffraction peaks, and the rocking curves were measured for the (008) peak. The value of $c$ in the bulk LSCO, $c_{\text {bulk }}$, is equal to $13.232 \AA$. Films grow with different c's, as shown in Fig. 1a, where we plot (008) peaks for both $K_{\alpha_{1}}$ and $K_{\alpha_{2}}$ radiation for several films. The peak is observed at the largest angle in film $A$. It is gradually shifted to smaller $2 \Theta$ on going to film $G$, indicating the increase in $c$. In addition, the peaks broaden on going from $A$ to $G$, suggesting that there is substantial distribution of the $c$-values $(\Delta c)$ in samples with large $c$, resulting from some structural disorder. This disorder cannot be caused by oxygen vacancies, because oxygen deficiency leads to a decrease in $c$-value, contrary to our results. A possible cause may be the gradual relief of strain across the thickness the film, which should contribute to the increase in the width of the rocking curve.
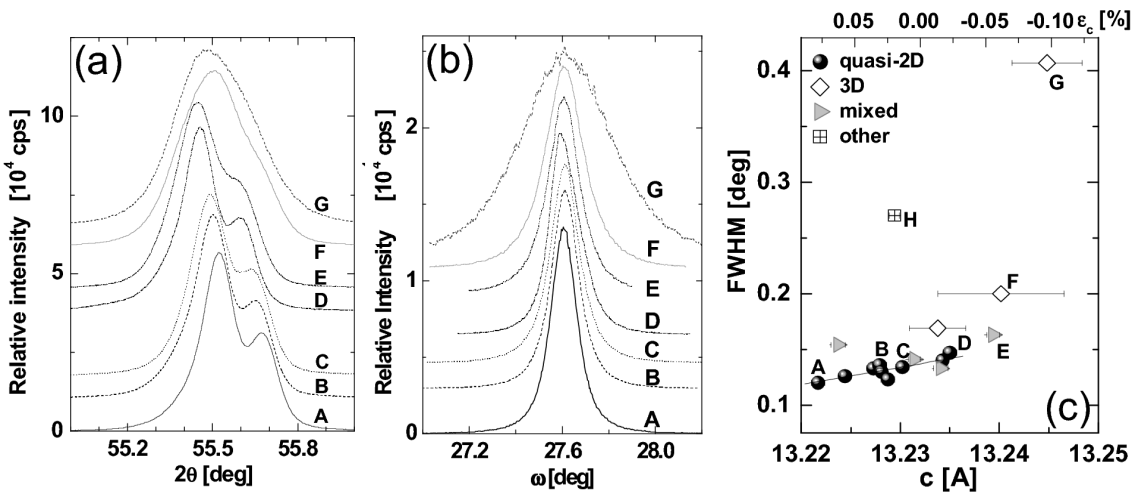

Fig. 1. The $\Theta-2 \Theta$ spectrum in the vicinity of (008) peak (a) and the rocking curves (b) for several LSCO films. The spectra are shifted for clarity. (c) FWHM versus $c$ for a larger batch of samples (samples $A-G$ are marked). Different symbols indicate films which grow in different fashion as described in the text.

Indeed, large $\Delta c$ is accompanied by the wide rocking curve (Fig. 1b). Figure $1 \mathrm{c}$ shows the correlation between the $c$ and the full width at half maximum (FWHM) of the rocking curve for a large batch of films. The errorbars on $c$ are standard deviations from the fit, reflecting the magnitude of $\Delta c$. The data are seen to follow a well-defined trend, on the basis of which we divide films into four groups (circles, diamonds, triangles, and crossed point). The first group consists of films in which the $\Delta c$ is very small $(<0.001 \AA)$, and the FWHM increases linearly with increasing $c$, but remains below $0.15 \mathrm{deg}$ (films $A$ to $D$ ). In the second group (diamonds) the FWHM starts to grow more abruptly and the $\Delta c$ is large $(F$ and $G$ ). The third group (triangles) contains films in which either $\Delta c$ or FWHM is somewhat enhanced in comparison with the first group. The correlation 
seen for these three groups strongly supports the conclusion that the increase in the $c$-value above a certain limit is associated with the gradual relief of strain. The exception is the film $H$ (crossed square), in which large FWHM is not accompanied by large $\Delta c$, suggesting that in this film the nature of the disorder is different. The top scale in Fig. 1c shows the strain defined as $\varepsilon_{c}=\left(c_{\text {bulk }}-c_{\text {film }}\right) / c_{\text {bulk }}$. The gradual strain relief appears in the films with a large negative $\varepsilon_{c}$. Using a 4-cycle diffractometer, we have verified that the $a$-axis (in-plane) lattice parameter increases linearly with the decrease in the $c$-axis parameter. This indicates that films grow either with the compressive in-plane strain (negative $\varepsilon_{c}$ ), or with the tensile in-plane strain (positive $\varepsilon_{c}$ ).
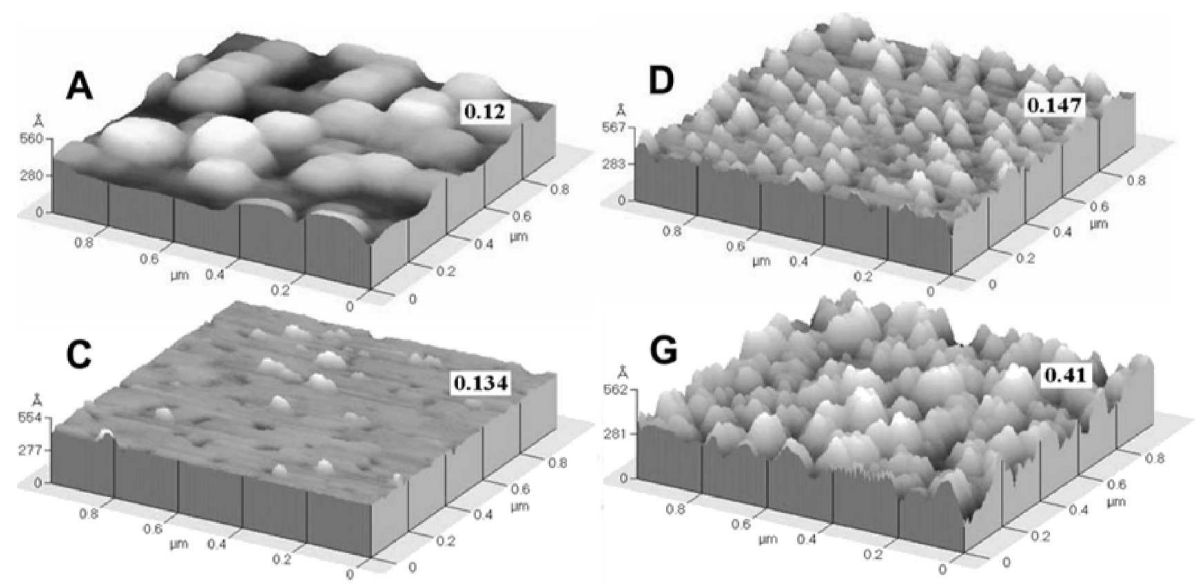

Fig. 2. AFM images of films $A, C, D$, and $G$. Image area is $1 \times 1 \mu \mathrm{m}^{2}$. The numbers indicate the FWHM for each film.

The AFM images of the film surface (Fig. 2) shows that the films with small tensile or compressive strain $(C$ and $D)$ are quite smooth, so that the growth is quasi-2D. The islands of substantial height (up to $10 \mathrm{~nm}$ ) are seen, but the areas between islands are filled and the grains are well fused. This is most likely a mixed type of growth, with the first layers growing in a layer-by-layer mode, followed by an island growth mode [5]. The increase in strain, either tensile (film $A$ ) or compressive (film $G$ ), produces rough films with remarkably different morphology. Film $G$ contains a collection of disordered grains of various sizes with conical shape. This suggests 3D growth, when new grains nucleate and grow rapidly before the previous layer of grains has a chance to coalesce. In contrast, the surface of $A$ film, with the largest tensile strain, consists of regularly shaped, pyramidal grains with flat tops, and with deep grooves between grains. The crystalline quality of the grains is excellent but they are weakly coupled. This is columnar growth mode, similar to the one observed during the growth of $\mathrm{LaCaMnO}_{3}$ on $\mathrm{MgO}$ substrate inducing tensile strain in the films [6]. 
The superconducting properties of LSCO films depend strongly on the film morphology, as will be discussed in detail elsewhere. Briefly, the quasi-2D films show high $T_{\mathrm{c}}$ and low residual resistivity. Large strain leads to broad superconducting transitions, either as a result of the gradual strain relief (compressive strain), or because of weak links (tensile strain).

The most interesting finding of this study is the observation of tensile strain built into some films during the growth. This is despite the fact that the deposition conditions were kept identical for all films. We do not have an explanation of this effect yet. It cannot be directly related to the substrate-induced strain, since the substrate in-plane lattice parameter is much smaller than the bulk in-plane parameters of the LSCO. This suggests that some unusual process occurs during the growth. The possibilities include, between others, the formation of precipitates or the off-stoichiometric layer in the initial stages of the growth, and/or the nucleation of dislocations. Further studies are either already in progress or are planned, using films with different thicknesses and transmission electron microscopy imaging.

\section{Conclusions}

The LSCO films on LSAO substrates are grown with different degrees of built-in strain, ranging from a large compressive to a large tensile in-plain strain. The strain affects morphology and the other properties of the films. The tensile strain cannot be simply attributed to a lattice parameter mismatch between the substrate and the film.

\section{Acknowledgment}

This work was supported by the State Committee for Scientific Research grant 2P 03B 04423.

\section{References}

[1] J.-P. Locquet, J. Perret, J. Fompeyerine, E. Machler, J.W. Seo, G. Van Tendeloo, Nature 394, 453 (1998).

[2] H. Sato, M. Naito, Physica C 274, 221 (1997); H. Sato, A. Tsukada, M. Naito, A. Matsuda, Phys. Rev. B 61, 12447 (2000); ibid. 62, R799 (2000).

[3] M.Z. Cieplak, A. Malinowski, K. Karpińska, S. Guha, A. Krickser, B. Kim, Q. Wu, C.H. Shang, M. Berkowski, P. Lindenfeld, Phys. Rev. B 65, 100504R (2002); A. Malinowski, M.Z. Cieplak, S. Guha, Q. Wu, B. Kim, A. Krickser, A. Perali, K. Karpińska, M. Berkowski, C.H. Shang, P. Lindenfeld, ibid. 66, 104512 (2002).

[4] M.Z. Cieplak, K. Karpińska, J. Domagała, E. Dynowska, M. Berkowski, A. Malinowski, S. Guha, M. Croft, P. Lindenfeld, Appl. Phys. Lett. 65, 3383 (1994); I.E. Trofimov, L.A. Johnson, K.V. Ramanujachary, S. Guha, M.G. Harrison, M. Greenblatt, M.Z. Cieplak, P. Lindenfeld, ibid. 65, 2481 (1994).

[5] E. Bauer, Z. Kristallogr. 110, 372 (1958).

[6] O.I. Lebedev, J. Verbeeck, G. Van Tendeloo, O. Shapoval, A. Belenchuk, V. Moshnyaga, B. Damashcke, K. Samwer, Phys. Rev. B 66, 104421 (2002). 